Article

\title{
Hybrid Vesicle Stability under Sterilisation and Preservation Processes Used in the Manufacture of Medicinal Formulations
}

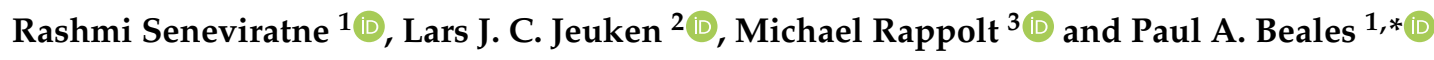 \\ 1 School of Chemistry, Astbury Centre for Structural Molecular Biology and Bragg Centre for Materials \\ Research, University of Leeds, Leeds LS2 9JT, UK; cm12rhs@leeds.ac.uk \\ 2 School of Biomedical Sciences, Astbury Centre for Structural Molecular Biology and Bragg Centre for \\ Materials Research, University of Leeds, Leeds LS2 9JT, UK; L.J.C.Jeuken@leeds.ac.uk \\ 3 School of Food Science and Nutrition and Bragg Centre for Materials Research, University of Leeds, Leeds \\ LS2 9JT, UK; M.Rappolt@leeds.ac.uk \\ * Correspondence: p.a.beales@leeds.ac.uk
}

Received: 6 March 2020; Accepted: 9 April 2020; Published: 15 April 2020

\begin{abstract}
Sterilisation and preservation of vesicle formulations are important considerations for their viable manufacture for industry applications, particular those intended for medicinal use. Here, we undertake an initial investigation of the stability of hybrid lipid-block copolymer vesicles to common sterilisation and preservation processes, with particular interest in how the block copolymer component might tune vesicle stability. We investigate two sizes of polybutadiene-block-poly(ethylene oxide) polymers $\left(\mathrm{PBd}_{12}-\mathrm{PEO}_{11}\right.$ and $\left.\mathrm{PBd}_{22}-\mathrm{PEO}_{14}\right)$ mixed with the phospholipid 1-palmitoyl-2-oleoyl-sn-glycero-3-phosphocholine (POPC) considering the encapsulation stability of a fluorescent cargo and the colloidal stability of vesicle size distributions. We find that autoclaving and lyophilisation cause complete loss of encapsulation stability under the conditions studied here. Filtering through $200 \mathrm{~nm}$ pores appears to be viable for sterilisation for all vesicle compositions with comparatively low release of encapsulated cargo, even for vesicle size distributions which extend beyond the $200 \mathrm{~nm}$ filter pore size. Freeze-thaw of vesicles also shows promise for the preservation of hybrid vesicles with high block copolymer content. We discuss the process stability of hybrid vesicles in terms of the complex mechanical interplay between bending resistance, stretching elasticity and lysis strain of these membranes and propose strategies for future work to further enhance the process stability of these vesicle formulations.
\end{abstract}

Keywords: liposomes; polymersomes; filtration; freeze-thaw; membrane mechanics; encapsulation stability

\section{Introduction}

Hybrid vesicles aim to combine the properties of biomimetic liposomes and synthetic polymersomes into composite membrane-bound capsules with broader tuneability of material properties [1-10]. This expanded material parameter space is anticipated to be beneficial in the development of hollow vesicle structures for technological applications such as sensors, biotechnology, nanoreactors, synthetic biology and formulated products, including those intended for medicinal purposes [11-21].

Individually, liposomes and polymersomes offer different advantages [22]. Liposomes, composed of natural lipids, form bilayer membranes that closely mimic the structural matrix of native biomembranes. This makes them highly biocompatible and provides a native-like environment if integral membrane proteins are desired to add functionality to the membrane [23-27]. However, 
liposomes can have inherent instabilities. Their membranes are highly flexible under bending deformations, but weak under stretching deformations, with a lysis strain of less than $5 \%[28,29]$. The labile fluidity of the membrane can lead to transient membrane defects that frustrates long term encapsulation stability and lipid peroxidation can cause chemical instabilities in these structures.

Polymersomes, due to their synthetic nature, are often less biocompatible than their lipid counterparts but offer greater mechanical stability and a broader chemical parameter space [30]. Amphiphilic copolymers that form vesicles can have several different architectures, the most common being $\mathrm{AB}$ diblock ( $\mathrm{A}=$ hydrophilic, $\mathrm{B}=$ hydrophobic) [6], $\mathrm{ABA}$ [31] and $\mathrm{ABC}$ tri-block polymers [32] where $A$ and $C$ are chemically different hydrophilic blocks and finally graft copolymers [33]. Formation of polymersomes depends on the amphiphilic co-polymers molecular weight, polydispersity and hydrophilic/hydrophobic block lengths ratio, which is ideally less than 1:3 to form vesicular structures [34]. Their membranes are often thicker than liposome membranes, which provides greater bending resistance and their enhanced elasticity under stretching makes them tough and durable [22]. The polymer chemistry can be designed to minimise chemical instability but also to incorporate novel functionality. In blending liposomes and polymersomes to create hybrid vesicles, the ambition is to combine advantageous properties of these materials while off-setting their weaknesses [35].

For medicinal applications in particular, stringent regulatory requirements exist for the sterilisation and preservation during the transportation and storage of these formulations [36]. Vesicles need to exhibit good stability under appropriate processing conditions that maintain the encapsulation of active compounds and the colloidal stability of vesicle structures.

Sterilisation is required to make the product safe for public use. It is the process by which all forms of life are destroyed, removed or permanently inactivated. Instead of an absolute measure of sterilisation, pharmaceutical industries use processes that reduce the probability of the survival of undesired organisms to a negligible level. Currently, pharmaceutical companies use thermal, filtration and irradiation techniques to sterilise their products [36,37]. Thermal sterilisation (Figure 1a), by using an autoclave, is the most common and the most reliable technique as it achieves destruction of microorganisms by irreversible denaturation of enzymes [36] and it allows the sterilisation of larger objects more easily than filtration [38].

a)

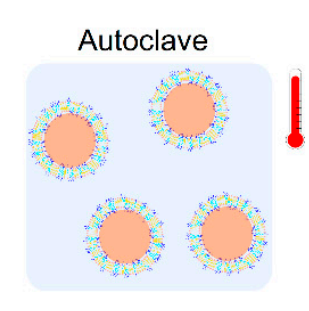

b)

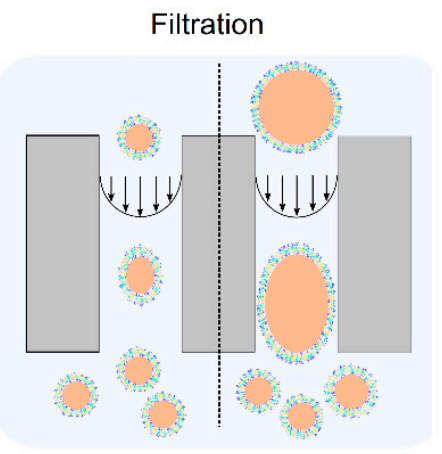

c)

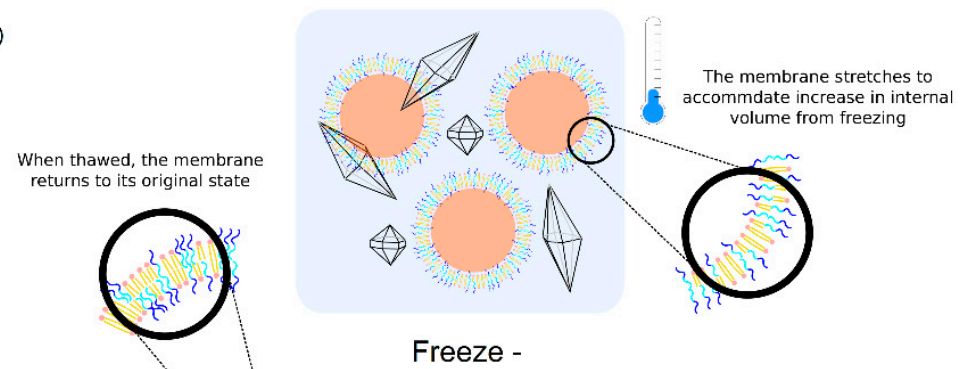

Figure 1. Schematic of sterilisation and preservation processes applied to vesicle formulations. (a) Autoclaving; (b) filtration; (c) freeze-thaw and lyophilisation. 
Filtration through a maximum of a $0.22 \mu \mathrm{m}$ membrane is usually used for sterilisation of thermally labile solutions, while sterilisation of active ingredients or medical devices can be achieved by irradiation [37]. Filtration exerts fluid shear stresses on vesicles as they pass down channels in the filter membrane (Figure 1b). While vesicles smaller than the filter pore size might fit within the channel without needing to deform, under flow, the Poiseuille velocity flow field in the channel indicates the fluid is moving much faster in the centre of the pore channel than at the edges. This will deform even small vesicles and, depending on the magnitude of these stresses, could induce transient pore formation in the membrane, compromising encapsulation stability, or even causing break-up of the vesicles. For larger vesicles, greater in size than the pore diameter, these vesicles must also deform at the entrance to the channel and will experience greater shear stresses that increases the probability that these vesicles might break-up into smaller structures.

Stability during the storage and transportation of vesicle formulations can also be a challenge. This may be in the transport between primary and secondary manufacturing sites or between the manufacturer and end-user. Once reaching the end-user, formulations may be stored for some time before their final use. Cold chain transportation is frequently used for medicinal products to maintain the stability and activity of their ingredients. Cryogenic conditions are frequently used for preservation during the transportation and storage of delicate biologics and labile formulations.

Liquid formulations are often frozen for preservation (Figure 1c). However, storing frozen samples for an extended period can cause ice crystal formation that could cause membrane damage for vesicles during freezing and thawing [39]. Besides potential damage from ice crystals, vesicles will become stressed by the expansion of water inside their lumen upon freezing. The volume of water expands by $\sim 9 \%$ upon freezing, which will exert a $\sim 6 \%$ areal strain on the confining membrane, which exceeds the lysis strain for lipid membranes. Indeed, freeze-thaw cycles are used to intentionally rupture liposomes during the passive loading of compounds from the bulk medium. Formulations may experience several freeze-thaw processes during manufacture, transport and storage, whether deliberate or unintentional. Fluctuations in temperature during transport from improper storage and shipping can cause some samples to reach their destination past their shelf life. This makes some drug formulations ineffective or even harmful [40,41].

Lyophilisation (freeze-drying) can also be used to maintain the stability of lipids during transportation and storage. During the freeze-drying process the product is frozen, thus immobilising the sample and allowing it to retain its original form, and then the water is removed by sublimation (Figure 1c), preventing microbial growth [42]. For lipids, the absence of water would minimise the rate of hydrolysis during storage [43]. However lyophilisation of liposome formulations is challenging to maintain encapsulation and structural stability. Additives are required for liposome cryopreservation, yet their efficacy can be variable and inconsistent [44].

Here, we investigate hybrid vesicles composed of a phospholipid 1-palmitoyl-2-oleoyl-snglycero-3-phosphocholine (POPC) and one of two amphiphilic polybutadiene-block-polyethylene oxide (PBd- $b$-PEO) diblock copolymers of different molecular weights under conditions designed to mimic industrial sterilisation and storage/transportation processes. We explore the full hybrid compositional space from pure lipid to pure polymer vesicles. Sterilisation is mimicked through autoclaving or multiple filtration passes through a filter with a $0.2 \mu \mathrm{m}$ pore size. The storage/transportation of samples is emulated through either lyophilisation and rehydration, or multiple freeze-thaw-vortex (FTV) cycles. This preliminary study of hybrid vesicle stability under these conditions is designed as a stringent test of their stability that will highlight advantages that can be gained through the composition of the hybrid formulation and to uncover aspects of these formulations that will require optimisation for future commercial translation. As such, we investigate the hybrid vesicle in a physiological saline buffer without additional additives for enhanced stability, such as potential cryo-preservatives. 


\section{Materials and Methods}

\subsection{Materials}

The di-block copolymers poly(1,2-butadiene)-block-(polyethylene oxide) (PBd-b-PEO) with total molecular weights of $1800 \mathrm{gmol}^{-1}$ and $1150 \mathrm{gmol}^{-1}$ were purchased from Polymer Source, Inc. (Montreal, Canada). $\mathrm{PBd}_{22}-\mathrm{PEO}_{14}$ (PDI 1.01) has a hydrophobic PBd block of $1200 \mathrm{gmol}^{-1}$ (>85\% 1,2 addition) and a hydrophilic PEO block of $600 \mathrm{gmol}^{-1}$ while polybutadiene-block-poly(ethylene oxide) $\left(\mathrm{PBd}_{12}-\mathrm{PEO}_{11}\right)$ (PDI 1.09) purchased from Polymer Source (Dorval, Montreal, Canada) has a hydrophobic PBd block of $650 \mathrm{gmol}^{-1}$ (85\% 1,2 addition) and a hydrophilic PEO block of $500 \mathrm{gmol}^{-1}$.

The lipid 1-palmitoyl-2-oleoyl-sn-glycero-3-phosphocholine (POPC) was purchased from Avanti Polar Lipids (Alabaster, AL, USA). 5(6)-carboxyfluorescein (CF) and other reagents were purchased from Sigma Aldrich (St. Louis, MO, USA).

\subsection{Vesicle Preparation}

Large unilamellar vesicles (LUVs) were prepared by the thin film rehydration and extrusion method. To generate different hybrid vesicle compositions, relative volumes of POPC (32 mM) and PBd-PEO (6.57 $\mathrm{mM})$ in chloroform were measured using a Hamilton syringe into a glass vial. The solutions were dried in a vacuum desiccator to give a lipid/polymer film and then rehydrated with $1.0 \mathrm{~mL}$ of aqueous solution of $60 \mathrm{mM} \mathrm{CF}$ with $40 \mathrm{mM}$ 4-(2-hydroxyethyl)-1-piperazineethanesulfonic acid (HEPES) and $20 \mathrm{mM}$ sodium chloride, adjusted the $\mathrm{pH}$ to 7.4 by dropwise addition of sodium hydroxide. The films were incubated at $50^{\circ} \mathrm{C}$ for $5 \mathrm{~min}$ and vortexed for $1 \mathrm{~min}$. The suspensions were then frozen in liquid nitrogen, thawed in a $60^{\circ} \mathrm{C}$ water bath and vortexed for $10 \mathrm{~s}$. This cycle was repeated 5 times. Suspensions were extruded 11 times though a 100 or $400 \mathrm{~nm}$ pore size polycarbonate membrane filters using a LiposoFast Basic Extruder. The nanovesicle samples were run on a Sephadex G50 column under gravity using $40 \mathrm{mM}$ HEPES and $20 \mathrm{mM}$ sodium chloride buffered to $\mathrm{pH} 7.4$ as the mobile phase to remove unencapsulated $\mathrm{CF}$ dye. The resulting $3 \mathrm{~mL}$ fractions were characterised using dynamic light scattering (DLS) for particle size distribution to confirm the presence of vesicles. The hybrid vesicles are then further analysed for stability under sterilisation and preservation processes.

\subsection{CF Release Assay}

The $100 \mathrm{~nm}$ sized hybrid vesicle samples were split into 4 fractions of $500 \mu \mathrm{L}(\sim 2 \mathrm{mM})$. Fraction 1 was thermally sterilised using an autoclave at $121^{\circ} \mathrm{C}$ for $15 \mathrm{~min}$. Fraction 2 was lyophilised using a VirTis Benchtop Pro Lyophiliser (Wolf Laboratories Ltd., York, UK) for $24 \mathrm{~h}$ after freezing the sample in nitrogen. Fraction 3 underwent 5 filtration cycles through a $13 \mathrm{~mm}$ PTFE $200 \mathrm{~nm}$ syringe filter device (Fisher Scientific Ltd., Hampton, NH, USA) with polypropylene housing. Fraction 4 was frozen in liquid nitrogen, thawed in a water bath at $60{ }^{\circ} \mathrm{C}$ and vortexed for $3 \mathrm{~s}$. The samples undergo 4 of these freeze-thaw-vortex (FTV) cycles.

The $400 \mathrm{~nm}$ sized vesicles were filtered through a $13 \mathrm{~mm}$ PTFE $200 \mathrm{~nm}$ syringe filter device (Fisher Scientific Ltd., Hampton, NH, USA) with a polypropylene housing 5 times.

$\mathrm{CF}$ is self-quenching at high concentrations $(>40 \mathrm{mM})[45,46]$, so the emission intensity at this concentration was often very low. The CF was encapsulated at $60 \mathrm{mM}$, so the control samples would have a low emission intensity. When the vesicles release the encapsulated $C F$, the $C F$ was diluted by the external buffer and the fluorescence intensity increased.

To measure the $\mathrm{CF}$ release, $0.5 \mathrm{~mL}$ fractions were diluted to $2 \mathrm{~mL}(\sim 18 \mu \mathrm{M})$ and the fluorescence emission at $519 \mathrm{~nm}$ of CF-encapsulated vesicles was measured with excitation set to $492 \mathrm{~nm}$ using a Horiba Scientific FluoroMax Plus (Horiba Ltd., Kyoto, Japan). Measurements were made on initial vesicle fractions and between every processing cycle for each fraction. The initial vesicle preparations were ruptured with $50 \mu \mathrm{L}$ of $10 \%(w / v)$ Triton X-100 (end concentration $0.91 \%(w / v)$ ) (Scientific Laboratory Supplies Ltd., Nottingham, UK) to completely destabilise the vesicles and release the encapsulated CF before a final fluorescence emission at $519 \mathrm{~nm}$ was measured. 
The CF \% release was calculated by

$$
\% \text { CF release }=\frac{I_{i}-I_{0}}{I_{t}-I_{0}} \times 100 \%
$$

where $I_{0}$ is the initial intensity of the sample, $I_{i}$ is the intensity of the sample after each processing cycle (from autoclaving, lyophilisation, FTV or filtering), and $I_{t}$ is the intensity after the initial sample is burst with Triton X-100.

\subsection{Dynamic Light Scattering (DLS)}

After separating hybrid vesicles with encapsulated CF from excess CF, the vesicles were characterised using a Malvern Zetasizer Nano ZSP (Malvern Panalytical Ltd., Malvern, UK) with scattering angle $173^{\circ}$ to determine their size. Each hybrid vesicle sample $(0.5 \mathrm{mM})$ was measured at $25{ }^{\circ} \mathrm{C}$ by the DLS and the results from 3 independent repeats were averaged. Size distributions were also measured after the processing steps described above to assess the colloidal stability of these vesicle formulations under these conditions.

\subsection{Cryo-Electron Microscopy (cryo-EM)}

Vesicles of $50 \mathrm{~mol} \% \mathrm{PBd}_{22}-\mathrm{PEO}_{14}$ at 100 and $400 \mathrm{~nm}$ were imaged after initial preparation and after the final freeze-thaw-vortex and filtration cycles respectively. For $100 \mathrm{~nm}$ vesicles, $400 \mathrm{mesh}$ 2/2 $\mu \mathrm{m} \mathrm{Cu}$ Quantifoil grids were used, while for $400 \mathrm{~nm}$ vesicles, 400 mesh Cu Lacey grids were used. All the carbon-coated grids were glow discharged for $33 \mathrm{~s}$ at $10 \mathrm{~mA}$ to render the surface hydrophilic. Samples $(3 \mu \mathrm{L}, \sim 2 \mathrm{mM}$ ) were placed directly onto the grid with a hold time of $40 \mathrm{~s}$, using a FEI Vitrobot mkIV, using a blotting time of $6 \mathrm{~s}$ and a blot force of 6 . The samples were kept in closed cryo-pucks under liquid nitrogen until required.

To image the girds, an FEI Titan KRIOS microscope (Thermofisher, Waltham, MA, USA) with an accelerating voltage of $300 \mathrm{kV}$ was used with a defocussing of $3 \mu \mathrm{m}$ at a magnification of $\times 130,000$ and $\times 26,000$. The resolution for these images was 0.11 and $0.55 \mathrm{~nm} /$ pixel, respectively.

Quantitative analysis of the images required taking diameter measurements and observations of all the vesicles in the grid holes of the carbon-coated copper grid using ImageJ. Histograms were made to document the size distribution observed in the grid holes, and notes were made on the ratio of multilamellar vesicles (MLVs) to unilamellar vesicles (ULVs).

\section{Results}

We investigated the stability of hybrid vesicles under conditions designed to mimic industrial sterilisation and preservation processes. Our hybrid vesicles were composed of the lipid POPC blended with one of two PBd- $b$-PEO diblock copolymers of different length: $\mathrm{PBd}_{22}-\mathrm{PEO}_{14}$ or $\mathrm{PBd}_{12}-\mathrm{PEO}_{11}$. We explored the full compositional parameter space from pure lipid to pure polymer vesicle compositions. Hybrid vesicles were formed by the thin film rehydration and extrusion method. The vesicles were loaded with the hydrophilic fluorophore CF as a model encapsulated compound. We studied the encapsulation stability through release of CF from vesicles by fluorescence spectroscopy and the colloidal stability of the vesicle formulations from their hydrodynamic size distributions obtained by DLS.

\subsection{Autoclaving and Lyophilisation}

Autoclaving and lyophilisation of hybrid vesicles would be the preferred choices for sterilisation and preservation, if these formulations can be made to be stable under these conditions. Unfortunately, both processes proved to be too destructive, causing vesicles to become unstable and release their entire contents. Only vesicles composed of the pure components were investigated: 100\% POPC, 100\% 
$\mathrm{PBd}_{22}-\mathrm{PEO}_{14}$ and $100 \% \mathrm{PBd}_{12}-\mathrm{PEO}_{11}$. As $100 \% \mathrm{CF}$ release was observed in all cases (Figure 2a), it was considered extremely unlikely that hybrid blends would perform any better.
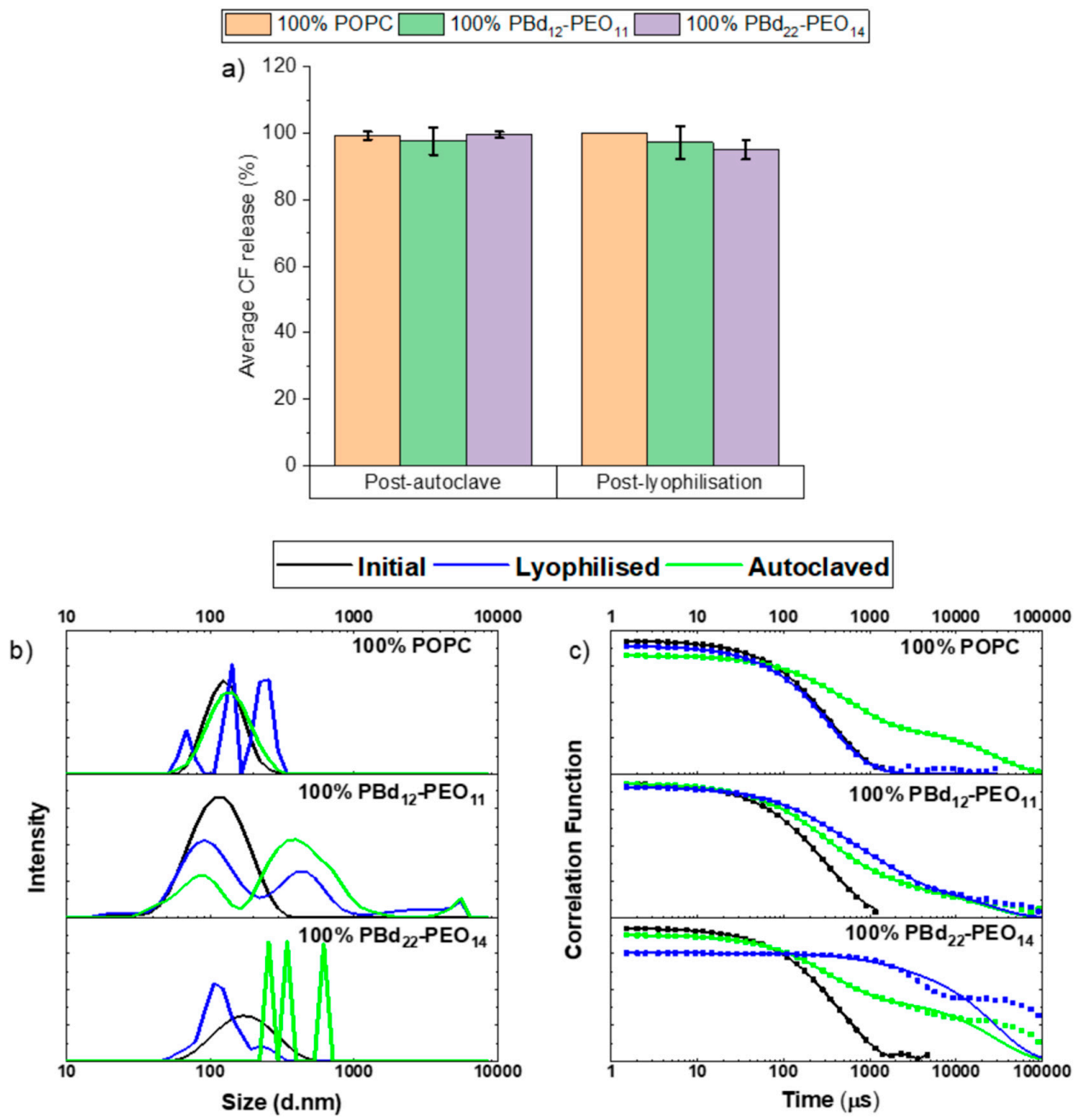

Figure 2. Release of encapsulated 5(6)-carboxyfluorescein (CF) and size distributions from vesicles following autoclaving or lyophilisation. (a) Vesicle compositions 100\% 1-palmitoyl-2-oleoylsn-glycero-3-phosphocholine (POPC), 100\% polybutadiene-block-poly(ethylene oxide) $\left(\mathrm{PBd}_{12}-\mathrm{PEO}_{11}\right)$ and $100 \% \mathrm{PBd}_{22}-\mathrm{PEO}_{14}$ were investigated following autoclaving or lyophilisation. Each measurement was performed in triplicate and the errors data points represent mean \pm s.d. The dynamic light scattering (DLS) distributions of hydrodynamic diameters from (b) constrained regularisation method for inverting data (CONTIN) fits size distributions and (c) the fitted autocorrelation functions. The size distributions shown represent the average distribution from three independent repeats.

The colloidal stability of the vesicles under these conditions was similarly poor. DLS size distributions were collected before and after autoclaving and lyophilisation (Figure 2b). The fitting software returned errors for all post-process samples except for autoclaving of $100 \%$ liposomes. The poor fits to the auto-correlation function in these cases can be seen in Figure 2c. The delayed exponential decay shown in the auto-correlation functions is interpreted as aggregation and/or structural instabilities of the vesicles during autoclaving or lyophilisation. The fact that the autocorrelation function does not reach the baseline in some cases indicates the presence of larger multimicron structures that are likely to undergo sedimentation.

Since these vesicles are unstable under autoclaving and lyophilisation, we further investigate other potential methods of sterilisation and preservation. However, we do not conclude that these techniques are fundamentally hopeless for use with hybrid vesicles: further development of the vesicle 
formulations would be required; for example, additional additives to the solution environment that help protect the integrity of the vesicles undergoing these processes.

\subsection{Filtration}

Sterilisation can be achieved by filtrations through a membrane with a maximum pore size of $0.22 \mu \mathrm{m}$. Here, we use a $0.2 \mu \mathrm{m}$ pore size membrane to filter hybrid vesicles for between one and five cycles. Initially, we investigated hybrid vesicles between 0 and $100 \mathrm{~mol} \%$ block copolymer content in $25 \mathrm{~mol} \%$ increments for both PBd- $b$-PEO polymers. These vesicles were formed by extrusion through a $100 \mathrm{~nm}$ pore size membrane and encapsulated the $C F$ dye.

All vesicle compositions only exhibited minimal contents release across five filtration cycles, with the average contents release reaching at most $10 \%$. Figure 3a shows filtration-induced contents release from hybrid vesicles containing the shorter $\mathrm{PBd}_{12}-\mathrm{PEO}_{11}$ block copolymer. The unitary POPC and $\mathrm{PBd}_{12}-\mathrm{PEO}_{11}$ vesicles exhibit very low $(<5 \%)$ contents release across five filtration cycles and no vesicle composition exceeds $10 \%$ average release. Analysis of variance shows that there is no statistically significant $(\mathrm{p}<0.05)$ difference between the different vesicle compositions studied.
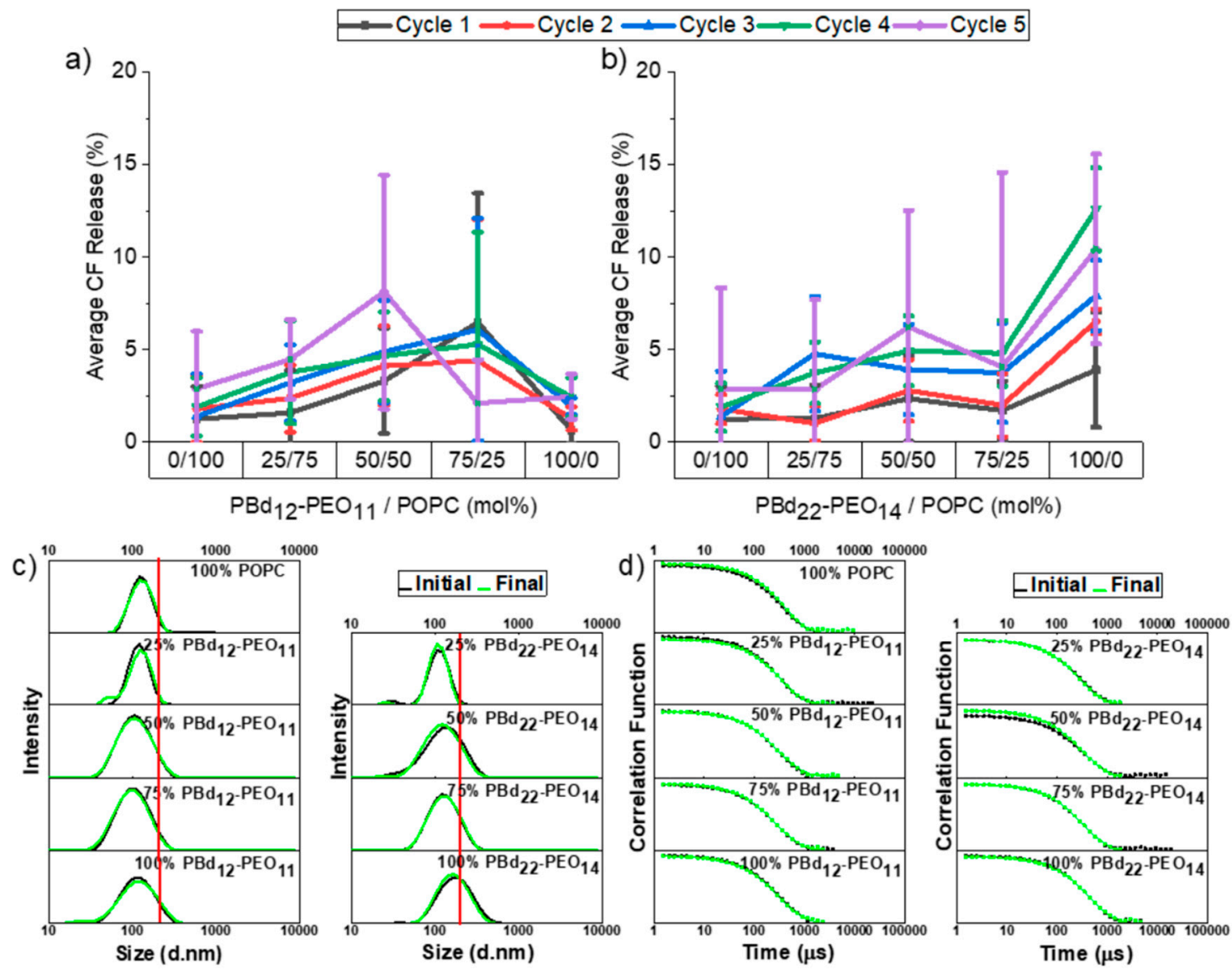

Figure 3. Release of encapsulated CF following filtration and size distributions before and after five filtration cycles. CF release $\%$ is plotted against membrane composition for hybrid vesicles composed of POPC and (a) $\mathrm{PBd}_{12}-\mathrm{PEO}_{11}$, or (b) $\mathrm{PBd}_{22}-\mathrm{PEO}_{14}$. Data are shown for between one and five filtration cycles through a $0.2 \mu \mathrm{m}$ pore size filter membrane. Each measurement was performed in triplicate and the errors data points represent mean \pm s.d. The DLS distributions of hydrodynamic diameters of $\mathrm{PBd}_{12}-\mathrm{PEO}_{11}$ or $\mathrm{PBd}_{22}-\mathrm{PEO}_{14}$ hybrid vesicles before and after five filtration cycles: (c) CONTIN fit size distributions; (d) the fitted autocorrelation functions. The size distributions shown represent the average distribution from three independent repeats. The line at $0.2 \mu \mathrm{m}$ denotes the filtration pore size. 
Perhaps surprisingly, the largest contents release occurred in vesicles composed of the larger $\mathrm{PBd}_{22}-\mathrm{PEO}_{14}$ block copolymer (Figure $3 \mathrm{~b}$ ), where a priori expectations might be that these would be the most mechanically stable vesicles. The only statistically significant difference in contents release is for pure $\mathrm{PBd}_{22}-\mathrm{PEO}_{14}$ polymersomes, which is significant when compared to all other membrane compositions, where the extent of release marginally exceeds $10 \%$ for the fourth and fifth filtration cycle. However, this contents release is still relatively low, even after several filtration cycles.

Hybrid vesicles ( $100 \mathrm{~nm}$ extrusion pore size) also demonstrate colloidal stability following multiple filtration cycles (Figure 3c,d and Tables S1-S4). Vesicle size distributions obtained by DLS before and after five filtration cycles were comparable for all hybrid vesicle compositions and both polymer sizes. No evidence of break-up or aggregation of vesicles was observed. The red line on these graphs denotes the filter pore size of $200 \mathrm{~nm}$. Vesicles with higher polymer content exhibit a tail in their size distribution which stretches beyond the filtration pore size. While these vesicles can pass the filter without notable changes in the particle size distribution, these also largely correlate with the vesicle compositions that exhibit the highest contents release, suggesting that the probability of transient pores forming in the vesicle membrane increases when vesicles are larger than the pore size and must significantly deform during the transit of the pore channel. This is particularly notable for the size distribution of $100 \mathrm{~mol} \% \mathrm{PBd}_{22}-\mathrm{PEO}_{14}$ vesicles, where the highest contents release was measured in Figure $3 b$.

Given that most of the $100 \mathrm{~nm}$ extruded vesicles are smaller than the $200 \mathrm{~nm}$ filter size, we presented a more stringent test of hybrid vesicle stability under filtration by using $400 \mathrm{~nm}$ extruded vesicles. In this series of experiments, we investigated the pure lipid and polymer vesicles as well as the $50 \mathrm{~mol} \%$ hybrid compositions by both polymer sizes.

Contents release from $400 \mathrm{~nm}$ extruded vesicles was significantly greater than previously observed for $100 \mathrm{~nm}$ extruded vesicles (Figure 4). While the shorter $\mathrm{PBd}_{12}-\mathrm{PEO}_{11}$ polymer appears to provide a mildly protective effect in hybrid vesicles with a gentle reduction in CF release observed with increasing polymer composition, these differences are not statistically significant (Figure 4a). The only statistically significant difference in contents release is again observed for pure $\mathrm{PBd}_{22}-\mathrm{PEO}_{14}$ polymer vesicles, which show reduced encapsulation stability compared to the POPC liposomes and $50 \mathrm{~mol} \%$ hybrid vesicles (Figure $4 \mathrm{~b}$ ). Despite this, overall contents release after five filtration cycles is small, being less than $15 \%$ for all compositions except the $100 \mathrm{~mol} \% \mathrm{PBd}_{22}-\mathrm{PEO}_{14}$ vesicles, where contents release exceeds $25 \%$ after five filter passes.

The filtration of $\mathrm{PBd}_{22}-\mathrm{PEO}_{14}$ vesicles was more difficult than other vesicle compositions, requiring much higher pressures to be applied to force the vesicle sample through the filter. This indicates that these vesicles are the most rigid due to the high bending stiffness of the thicker polymer membranes. The higher pressures that need to be applied during filtration are therefore the likely cause of the enhanced contents release from these vesicles. On the other hand, the shorter polymer has lower bending resistance, allowing these vesicles to more easily pass through the filter and may impart some enhanced elasticity to hybrid vesicles, giving a mild protective effect to hybrid vesicles under filtration.

All vesicles were much smaller in size than the $400 \mathrm{~nm}$ pore size of the extrusion membrane for vesicle preparation (Figure 4c,d and Tables S5-S8). However, a significant proportion of vesicles are larger than the $200 \mathrm{~nm}$ filter size, meaning that they would need to significantly deform or break-up to pass through the filter. The initial $100 \% \mathrm{PBd}_{22}-\mathrm{PEO}_{14}$ vesicle size distribution also showed a bimodal distribution with peaks at 108 and $519 \mathrm{~nm}$. Following five filter passes, all pure component vesicles, whether lipid or polymer, showed multimodal size distributions with larger structures present in solution that are indicative of aggregation and loss of colloidal stability in these samples. 
a)

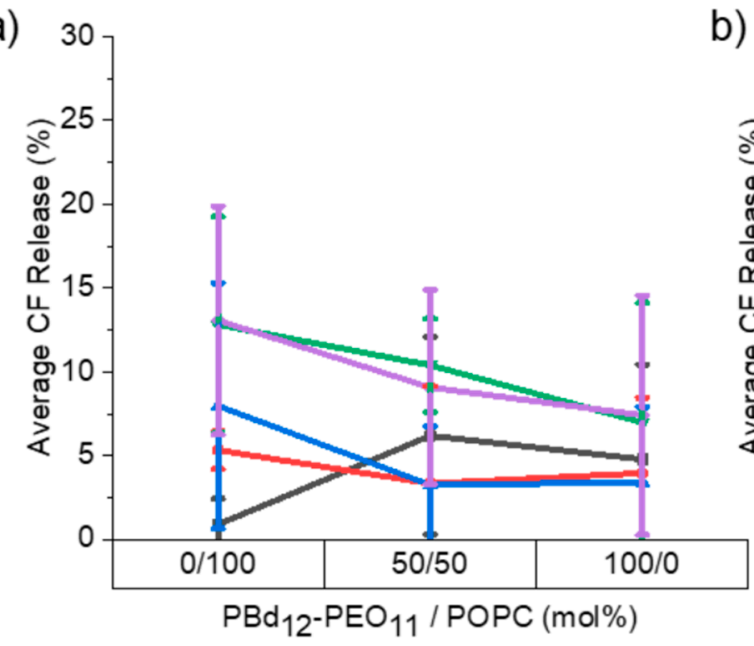

b) 30

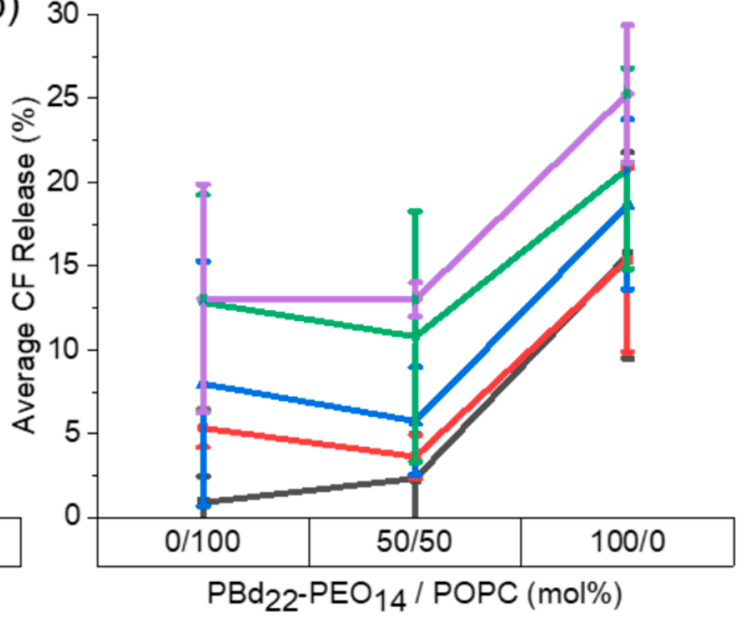

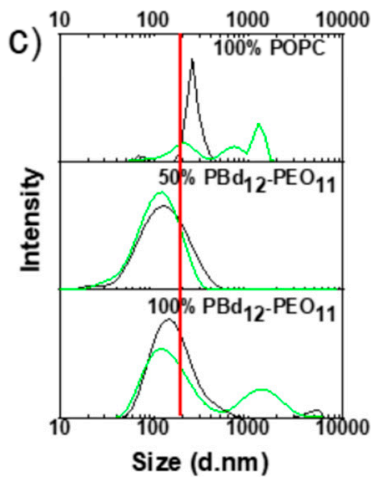
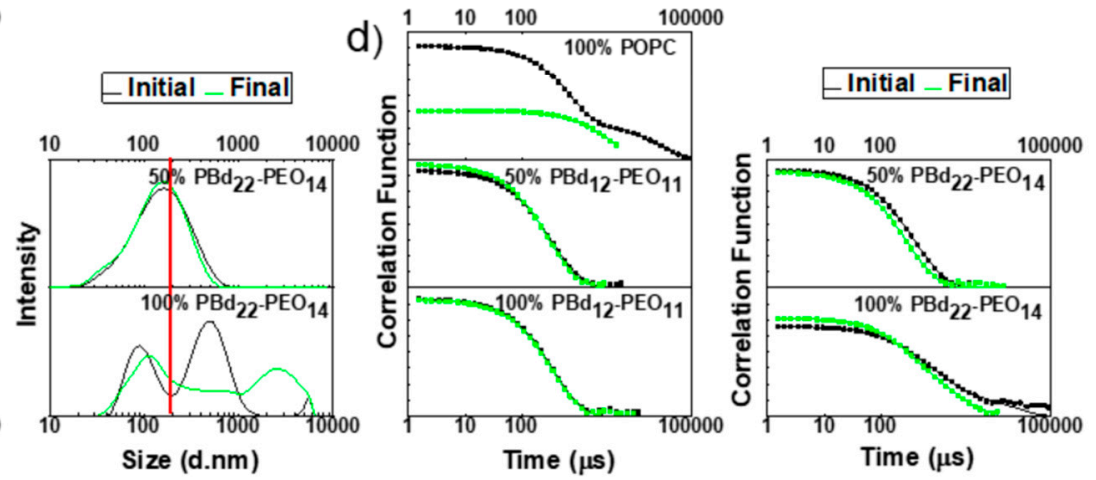

Figure 4. Release of encapsulated CF from $400 \mathrm{~nm}$ hybrid vesicles following filtration and size distributions before and after five filtration cycles. $\mathrm{CF}$ release $\%$ is plotted against membrane composition for hybrid vesicles composed of POPC and (a) $\mathrm{PBd}_{12}-\mathrm{PEO}_{11}$, or (b) $\mathrm{PBd}_{22}-\mathrm{PEO}_{14}$. Data are shown for between one and five filtration cycles through a $0.2 \mu \mathrm{m}$ pore size filter membrane. Each measurement was performed in triplicate and the errors data points represent mean \pm s.d. The DLS distributions of hydrodynamic diameters of $\mathrm{PBd}_{12}-\mathrm{PEO}_{11}$ or $\mathrm{PBd}_{22}-\mathrm{PEO}_{14}$ hybrid vesicles before and after five filtration cycles: (c) CONTIN fit size distributions; (d) the fitted autocorrelation functions. The size distributions shown represent the average distribution from three independent repeats. The line at $0.2 \mu \mathrm{m}$ denotes the filtration pore size.

Interestingly, both $50 \%$ hybrid vesicle formulations fared best under filtration with comparable monomodal size distributions before and after filtration. This enhanced colloidal stability might be due to the synergistically enhanced properties of the blended lipid-polymer hybrid membranes. However, it is also plausible that this is simply due to the smaller initial size distribution of these hybrid vesicles $\left(145 \mathrm{~nm}\right.$ for $50 / 50 \mathrm{~mol} \%$ POPC/PBd $\mathrm{Pd}_{12}-\mathrm{PEO}_{11}$ hybrid vesicles and $182 \mathrm{~nm}$ for $50 / 50 \mathrm{~mol} \%$ POPC/ $\mathrm{PBd}_{22}-\mathrm{PEO}_{14}$ hybrid vesicles). We previously observed good colloidal stability for $100 \mathrm{~nm}$ extrusion membrane vesicle formulations, therefore this latter explanation seems most likely. This smaller initial size distribution of $50 \mathrm{~mol} \%$ hybrid vesicles might be due to a preferred curvature of the hybrid vesicle membrane that limits the maximum size of vesicles that form at this composition.

We further investigated the $400 \mathrm{~nm} 50 / 50 \mathrm{~mol} \% \mathrm{POPC} / \mathrm{PBd}_{22}-\mathrm{PEO}_{14}$ hybrid vesicles before and after filtration by cryo-electron microscopy (cryo-EM) (Figure 5). For cryo-EM, an aqueous sample is placed on a carbon-coated copper grid which is plunged in liquid ethane that vitrifies the water into a glass-like state to enable the sample to be seen [47,48]. Ice thickness can vary depending on the dimensions of the particle but usually ranges from a few nanometres to a hundred nanometres [48]. 
The thicker the ice (>100 nm), the worse the resolution [49]. On the other hand, if the ice is too thin, then either the sample is pushed towards the edge of the grid holes, or the sample can have a high affinity for the carbon support, leaving the grid hole empty of sample and causing particle aggregation [47]. Variations in ice thickness can also sort the vesicles by size, concentrating the larger vesicles in thicker ice [50]. Lacey carbon-coated grids have a higher percentage of open area which allows larger particles to protrude over the edges of the grid holes, and therefore be seen at a higher resolution [51].
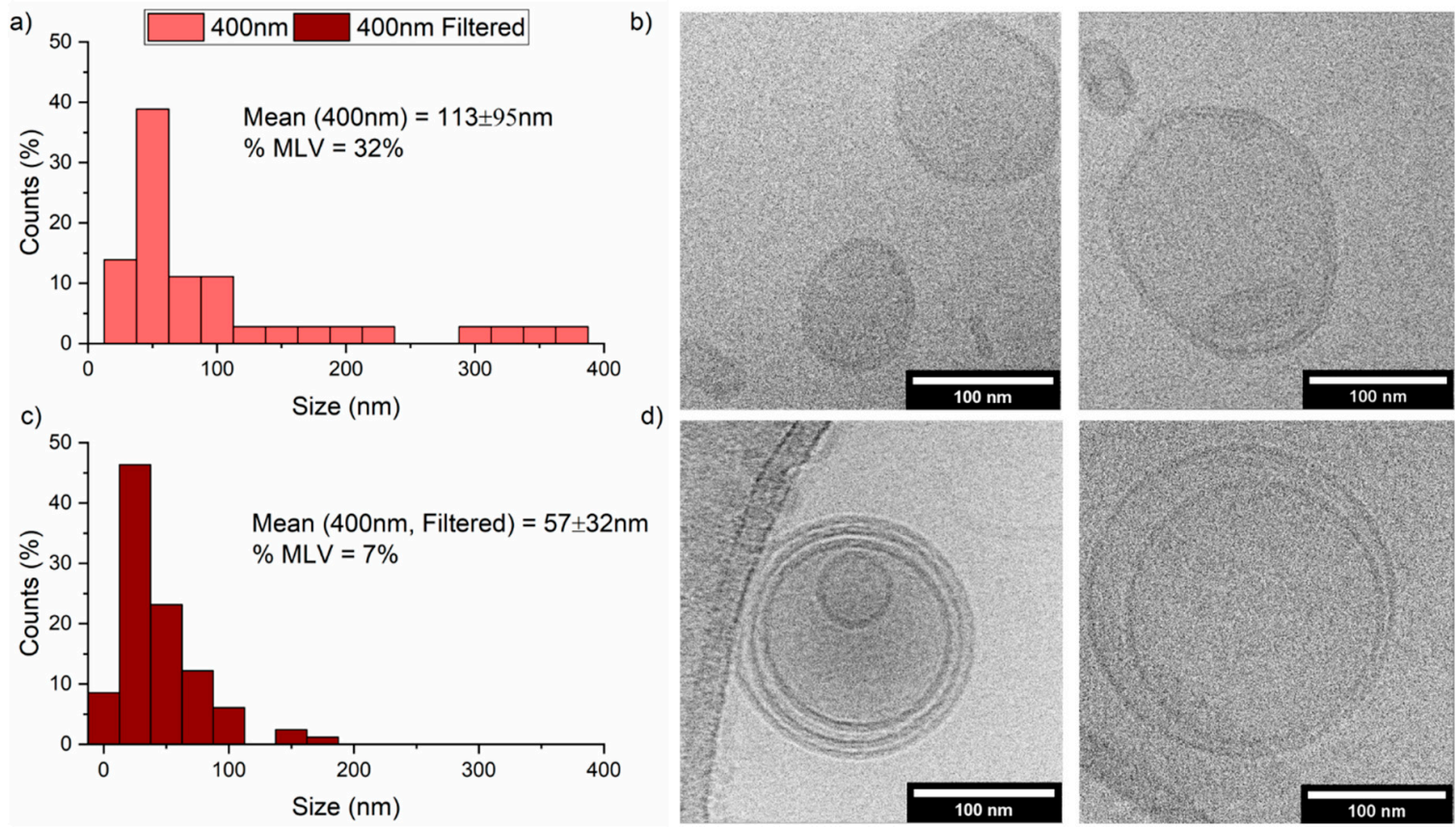

Figure 5. Cryo-transmission electron microscopy (Cryo-TEM) of $50 \mathrm{~mol} \% \mathrm{PBd}_{22}-\mathrm{PEO}_{14}$ hybrid vesicle (400 nm extrusion membrane) before and after five filtration cycles. (a) Histogram of initial vesicle sizes from cryo-TEM images; (b) representation images of vesicles before filtration; (c) histogram of vesicle sizes after filtration from cryo-TEM images; (d) representation images of vesicles after filtration. Scale bars represent $100 \mathrm{~nm}$.

Initial $50 \mathrm{~mol} \% \mathrm{PBd}_{22}-\mathrm{PEO}_{14}$ hybrid vesicles were found to have an average diameter of $113 \pm 95 \mathrm{~nm}$ and $32 \%$ of vesicles were observed to be multilamellar. This compares favourably with the previous DLS analysis where these vesicles have a z-average of $124 \mathrm{~nm}$ (PDI 0.279). However, following filtration, the cryo-EM analysis gave an average vesicle size of $57 \pm 32 \mathrm{~nm}$ with $7 \%$ of vesicles found to be multilamellar. This differs from our DLS analysis, which showed a post-filtration z-average of $120 \mathrm{~nm}$ (PDI 0.269). The reduction in average size observed in cryo-EM can be seen from the histograms to be due to the removal of larger $>200 \mathrm{~nm}$ vesicles from the post-filtered samples, likely due to vesicle break-up. Vesicle break-up during filtration would also explain the lower proportion of multilamellar vesicles that we observe. While larger vesicles can often be excluded from the thin ice in the holes of the EM grid, artificially leading to smaller size distributions by cryo-EM compared to DLS, the comparison between the pre- and post- filtered hybrid vesicles by cryo-EM would appear to be significant. The break-up of some larger and multilamellar vesicles during filtration would likely account for the $\sim 15 \%$ leakage of vesicle contents observed in Figure 4.

\subsection{Freeze-Thaw-Vortex}

An alternative preservation process to lyophilisation is to store vesicle samples frozen from the liquid state without sublimation of excess water. Therefore, we investigate the stability of vesicles to up to four freeze-thaw-vortex (FTV) cycles. The vortexing step following the thawing of frozen samples is included to ensure the vesicles are fully resuspended in the liquid state. 
Liposome encapsulation was observed to be highly unstable even after a single FTV cycle (Figure 6). This is unsurprising as freeze-thaw cycles are frequently used for passive loading of cargo in liposome preparation protocols, as this renders the membrane temporarily permeable. Conversely, $100 \%$ polymer vesicles exhibit much greater encapsulation stability across four FTV cycles with hybrid formulations showing increasing stability with increasing block copolymer composition in the membranes. For all vesicle compositions, CF release increased after each FTV cycle.

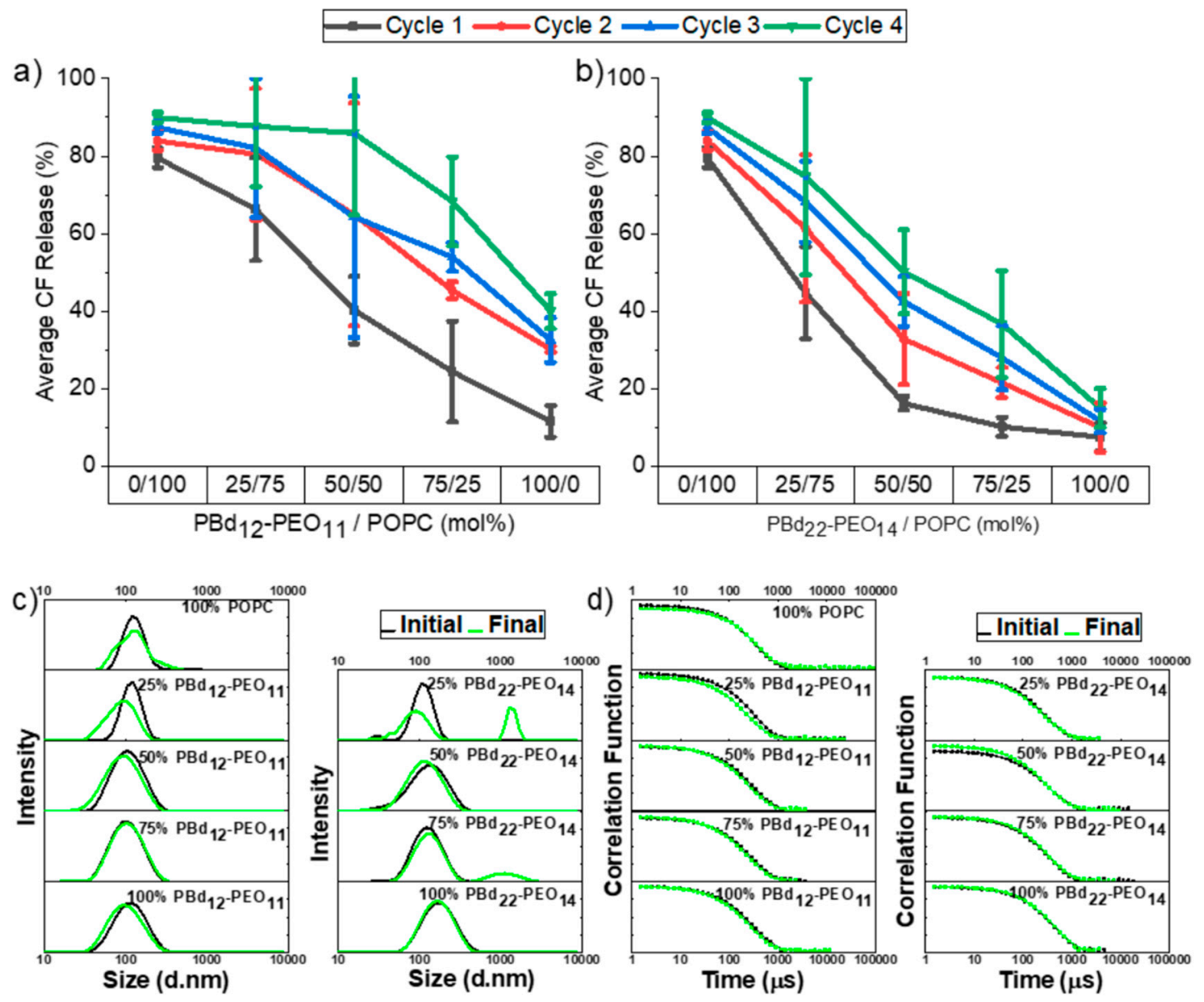

Figure 6. Release of encapsulated CF from $100 \mathrm{~nm}$ hybrid vesicles following freeze-thaw-vortex (FTV) cycles and size distributions before and after four FTV cycles. CF release $\%$ is plotted against membrane composition for hybrid vesicles composed of POPC and (a) $\mathrm{PBd}_{12}-\mathrm{PEO}_{11}$, or (b) $\mathrm{PBd}_{22}-\mathrm{PEO}_{14}$. Data are shown for between one and four FTV cycles. Each measurement was performed in triplicate and the errors data points represent mean \pm s.d. The DLS distributions of hydrodynamic diameters of $\mathrm{PBd}_{12}-\mathrm{PEO}_{11}$ or $\mathrm{PBd}_{22}-\mathrm{PEO}_{14}$ hybrid vesicles before and after four FTV cycles: (c) CONTIN fit size distributions; (d) the fitted autocorrelation functions. The size distributions shown represent the average distribution from three independent repeats.

Vesicles containing the larger $\mathrm{PBd}_{22}-\mathrm{PEO}_{14}$ polymer were also observed to have more stable $\mathrm{CF}$ encapsulation than those formulated with the $\mathrm{PBd}_{12}-\mathrm{PEO}_{11}$ polymer. We interpret this as the thicker membranes formed from the larger block copolymer having greater elasticity, permitting the membrane to stretch without rupture during the volume expansion of freezing. Furthermore, the thicker, more elastic membranes of these vesicles enhances protection from damage that might be caused by the local growth of ice crystals in the sample.

FTV cycles had the most impact on the colloidal stability of lipid-rich vesicles. We measured the vesicle size distributions by DLS before and after four FTV cycles (Figure 6c,d and Tables S9-S12). While the post-FTV size distributions are mostly monomodal (with the exception of $25 \mathrm{~mol} \%$ and 
$75 \mathrm{~mol} \% \mathrm{PBd}_{22}-\mathrm{PEO}_{14}$ hybrid vesicles), the size distributions for $100 \% \mathrm{POPC}$ and $25 \mathrm{~mol} \%$ polymer hybrid vesicles significantly broaden following four FTV cycles. In contrast, the size distributions of hybrid vesicles with $\geq 50 \mathrm{~mol} \%$ polymer content are broadly similar before and after the FTV cycles.

We further investigate the effect of freeze-thaw action on the size and morphology of $50 \mathrm{~mol} \%$ $\mathrm{PBd}_{22}-\mathrm{PEO}_{14}$ hybrid vesicles by cryo-EM. Quantitative analysis of the images shown in Figure 7 indicate that the vesicles have a similar size before and after the FTV cycles $(59 \pm 38$ vs. $60 \pm 24 \mathrm{~nm})$ and a slightly increased prevalence of multilamellar vesicles following FTV cycles (15\% vs. $24 \%$ ). However the cryo-EM grid preparation appears to favour smaller vesicles being in the grid holes, while the majority of the larger vesicles prefers to sit on the carbon-coated support as there is a large difference in size between the DLS and cryo-EM size distributions. The DLS analysis in Figure $6 c$ reports z-average sizes of $108 \mathrm{~nm}$ (PDI 0.213) and $101 \mathrm{~nm}$ (PDI 0.191) before and after four FTV cycles, respectively.
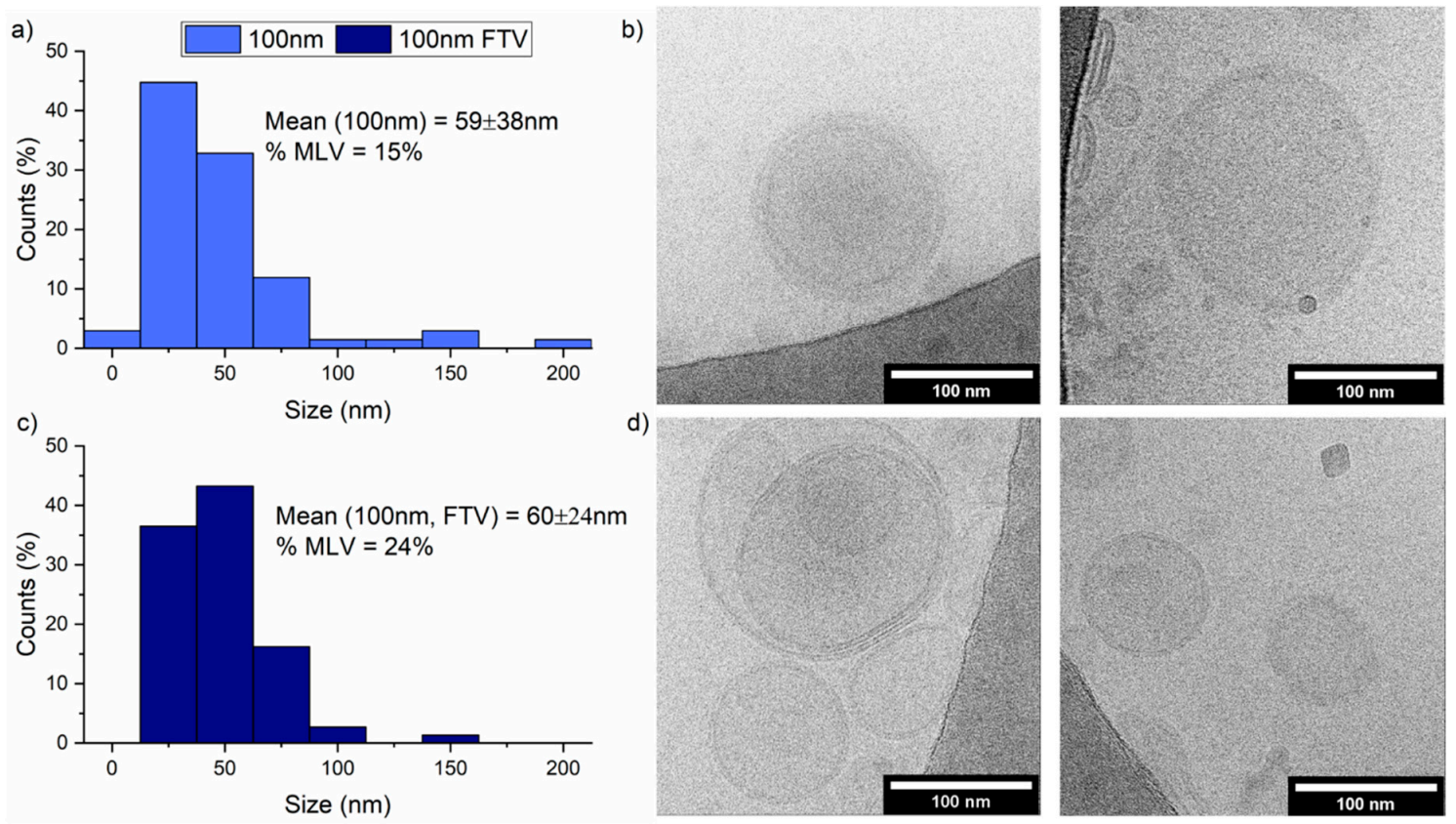

Figure 7. Cryo-TEM of $100 \mathrm{~nm} 50 \mathrm{~mol} \% \mathrm{PBd}_{22}-\mathrm{PEO}_{14}$ hybrid vesicles before and after four FTV cycles. (a) Histogram of initial vesicle sizes from cryo-TEM images; (b) representation images of vesicles before FTV; (c) histogram of vesicle sizes after FTV from cryo-TEM images; (d) representation images of vesicles after FTV. Scale bars represent $100 \mathrm{~nm}$.

\section{Discussion}

For vesicles composed of the pure lipid or block copolymer components, we find that sterilisation using an autoclave results in a loss of colloidal stability and the release of the encapsulated CF. Autoclaving involves exposure to high temperature, which is thought to be detrimental to lipid vesicles by causing aggregation [38,52]. The aggregation after heat sterilisation has been suggested to lead to instability in lipid vesicles by electrolytes, causing dehydration of the hydrophilic moiety [52]. However, in a previous study, it was found that egg or saturated lipid vesicles without encapsulated agents could be sterilised by autoclaving. When calcein or doxorubicin were encapsulated, however, the leakage was pronounced [38].

It was expected that the polymeric vesicles would be more resistant to heat exposure as polymer membranes are believed to be more robust than those formed by lipids alone. Polymersomes with a large membrane thickness ( $\mathrm{PEE}_{37}-\mathrm{PEO}_{40}$ and $\mathrm{PBd}_{46}-\mathrm{PEO}_{26}$ ) have previously been shown to have both encapsulation and colloidal stability to autoclave conditions, although there is a slight shift to a smaller vesicle size distribution [28]. To enhance the stability of the vesicles during heat sterilisation, 
it has been suggested that sugars or polyols could be used to stabilise the vesicles [52] and the drug could be encapsulated after vesicles have been autoclaved [38].

Lyophilisation also caused complete contents release and loss of colloidal stability in both pure lipid and pure PBd-b-PEO polymer vesicles, indicating rupture and aggregation of the vesicles. The initial freezing step could cause damage to vesicles from piercing by the ice crystals that form and expansion of the encapsulated aqueous volume during freezing. However, we later show that polymer-rich vesicle compositions in particular are comparatively stable under freeze-thaw action. Therefore, damage caused by freezing is not solely responsible for the chronic impact of lyophilisation on these vesicles.

During the second step of lyophilisation, water is removed by sublimation. As water is fundamentally essential to the self-assembly of amphiphiles into vesicle polymorphs, and the degree of hydration is known to facilitate phase transitions in lyotropic assemblies, it is perhaps not surprising that this dehydration step can cause severe structural instabilities. Here, we considered whether the enhanced stability of block copolymers alone might be enough to increase the stability of vesicles to lyophilisation; however, there is no evidence for this under the conditions currently tested. However, the damaging effects of lyophilisation have been minimised for liposomes by addition of cryoprotectants to the continuous aqueous phase. Small molecule cryoprotectants, such as glycerol or trehalose and other carbohydrates, can significantly reduce aggregation, fusion and leakage. Cryoprotectants have been successfully used to enhance the stability of liposomes to lyophilisation and rehydration [43]. However, this is notoriously challenging [44], and the potential for synergistic effects from cryoprotectants in the solution phase and block copolymers blended within hybrid vesicle membranes is a worthwhile avenue for future investigation.

While autoclaving and lyophilisation would need further investigation of added molecular protectants in the solvent environment to make them viable for hybrid vesicle processing, filtration and freeze-thaw processes appeared to be more immediately viable for sterilisation and preservation.

Vesicle filtration (200 nm pore size) resulted in relatively low contents release, particularly for vesicles formed by extrusion through a $100 \mathrm{~nm}$ membrane. These vesicles also showed good colloidal stability. However, this is perhaps relatively unsurprising since the DLS size distribution suggests that only a small proportion of vesicles are larger than the filter pore size, although hydrodynamic sheer stresses passing through a filter pore might cause minimal destabilisation of the membrane and loss of contents.

A more stringent test of vesicle stability under filtration was undertaken with vesicles formed by extrusion through $400 \mathrm{~nm}$ membranes. Now, a large proportion of vesicles are larger than the $200 \mathrm{~nm}$ filter pore size. More significant contents release is now observed (up to 25\%) and there is some loss of colloidal stability due to vesicle break-up and aggregation. Curiously, only $50 \mathrm{~mol} \%$ PBd- $b$-PEO hybrid vesicles maintained good colloidal stability, but this might be explained by the intrinsically smaller size distribution of these hybrid vesicles when initially formed.

When comparing between different vesicle compositions, it is interesting to note that the vesicle compositions with the highest filtration stability are perhaps initially non-intuitive. A reasonable initial hypothesis might be that vesicles with a high proportion of the larger PBd-b-PEO block copolymer would be more stable under filtration due to enhanced mechanical robustness from this polymer. However the converse is the case, where the worst performing vesicle composition is $100 \% \mathrm{PBd}_{22}-\mathrm{PEO}_{14}$. We interpret this to be due to the higher bending rigidity of the thicker membranes of these vesicles, offering higher resistance to deformations required to pass through the filter membrane. This is qualitatively manifested in the higher pressures that are required to force these vesicle samples across the filter membrane. These higher pressures likely have a more severe impact on encapsulation stability. Conversely, the smaller $\mathrm{PBd}_{12}-\mathrm{PEO}_{11}$ polymer does not have a significant impact on encapsulation stability, likely due to a similarly low bending rigidity to pure liposomes. These hybrid vesicles formed using this smaller polymer may also have enhanced membrane elasticity, imparting improved 
toughness to these vesicles, but this is not a statistically significant observation, likely due to the inherently low contents release that is already seen for the POPC liposomes.

Polymer-rich vesicles can also withstand several FTV cycles, making this a more viable alternative to lyophilisation for sample preservation. Lipid-rich vesicles have poor encapsulation stability under freeze-thaw action, but high polymer content likely enhances stability due to the increased stretching elasticity and higher lysis strain of polymersome membranes that is critical to their enhanced material toughness and durability. Now, membranes rich in the larger $\mathrm{PBd}_{22}-\mathrm{PEO}_{14}$ polymers present the best stability profile across several FTV cycles, having greater capacity to resist damage from solvent expansion stresses during freezing and the growth of ice crystals.

In summary, this initial investigation indicates that filtration and freeze-thaw are the most viable routes to sterilisation and preservation of hybrid vesicles while retaining good encapsulation and colloidal stability of these samples. A complex interplay between the bending rigidity, stretching elasticity and lysis strain of the membrane formulation determines which vesicles are most stable under each of these processes. While functional performance in the desired vesicle application will primarily dictate the optimisation of the membrane composition of vesicles, enhancing essential processing stability is a secondary consideration that cannot be completely ignored. While filtration is sufficient to sterilise small nano-vesicle samples, formulations that use larger vesicle sizes are less stable to this process and further efforts to develop autoclaving as a stable process for hybrid vesicles are worthwhile. Similarly, lyophilised vesicles would be favourable for frozen liquid samples for the preservation of vesicles for storage and transport to reduce economic costs and environmental impact. Therefore, further efforts to enhance hybrid vesicle stability to these harsher processes are still of significant interest. Furthermore, additional sterilisation processes might be considered, such as ultra-high temperature (UHT) and high temperature short time (HTST) [53] processing.

Supplementary Materials: The following are available online at http://www.mdpi.com/2073-4360/12/4/914/s1, Table S1: DLS fitting data of $\mathrm{PBd}_{12}-\mathrm{PEO}_{11}$ hybrid vesicles before five filtration cycles for Figure 3c, Table S2: DLS fitting data of $\mathrm{PBd}_{22}-\mathrm{PEO}_{14}$ hybrid vesicles before five filtration cycles for Figure 3c, Table S3: DLS fitting data of $\mathrm{PBd}_{12}-\mathrm{PEO}_{11}$ hybrid vesicles after five filtration cycles for Figure $3 \mathrm{c}$, Table S4: DLS fitting data of $\mathrm{PBd}_{22}-\mathrm{PEO}_{14}$ hybrid vesicles after five filtration cycles for Figure $3 c$, Table S5: DLS fitting data of $P_{B d} d_{12}-P_{11} O_{11}$ hybrid vesicles before five filtration cycles for Figure 4c, Table S6: DLS fitting data of $\mathrm{PBd}_{22}-\mathrm{PEO}_{14}$ hybrid vesicles before five filtration cycles for Figure 4c, Table S7: DLS fitting data of $\mathrm{PBd}_{12}-\mathrm{PEO}_{11}$ hybrid vesicles after five filtration cycles for Figure 4c, Table S8: DLS fitting data of $\mathrm{PBd}_{22}-\mathrm{PEO}_{14}$ hybrid vesicles after five filtration cycles for Figure $4 \mathrm{c}$, Table S9: DLS fitting data of $\mathrm{PBd}_{12}-\mathrm{PEO}_{11}$ hybrid vesicles before four FTV cycles for Figure 6c, Table S10: DLS fitting data of $\mathrm{PBd}_{22}-\mathrm{PEO}_{14}$ hybrid vesicles after four FTV cycles for Figure 6c, Table S11: DLS fitting data of $\mathrm{PBd}_{12}-\mathrm{PEO}_{11}$ hybrid vesicles after four FTV cycles for Figure 6c, Table S12: DLS fitting data of PBd $22-\mathrm{PEO}_{14} \mathrm{hybrid}$ vesicles after four FTV cycles for Figure 6c.

Author Contributions: Conceptualization, R.S. and P.A.B.; methodology, R.S., L.J.C.J., M.R. and P.A.B.; validation, R.S. and P.A.B.; formal analysis, R.S.; visualization, R.S.; investigation, R.S.; writing—original draft preparation, R.S. and P.A.B.; writing - review and editing, R.S., L.J.C.J., M.R. and P.A.B.; supervision, L.J.C.J., M.R. and P.A.B.; project administration, P.A.B.; funding acquisition, L.J.C.J., M.R. and P.A.B. All authors have read and agreed to the published version of the manuscript.

Funding: This research was funded by the Engineering and Physical Sciences Research Council (EPSRC) as part of the Centre for Doctoral Training in Soft Matter and Functional Interfaces (SOFI CDT), grant number EP/L015536/1.

Acknowledgments: We are grateful to Miranda Willis, Senior Process Technologist at GSK for the conversation with her that lead to this paper. We also thank Rebecca Thompson in the Astbury Biostructure Facility at the University of Leeds for support with cryo-electron microscopy.

Conflicts of Interest: The authors declare no conflict of interest.

\section{References}

1. Chemin, M.; Brun, P.M.; Lecommandoux, S.; Sandre, O.; Le Meins, J.F. Hybrid polymer/lipid vesicles: Fine control of the lipid and polymer distribution in the binary membrane. Soft Matter 2012, 8, 2867-2874. [CrossRef] 
2. Chen, D.; Santore, M.M. Hybrid copolymer-phospholipid vesicles: Phase separation resembling mixed phospholipid lamellae, but with mechanical stability and control. Soft Matter 2015, 11, 2617-2626. [CrossRef] [PubMed]

3. Dao, T.P.T.; Fernandes, F.; Ibarboure, E.; Ferji, K.; Prieto, M.; Sandre, O.; Le Meins, J.F. Modulation of phase separation at the micron scale and nanoscale in giant polymer/lipid hybrid unilamellar vesicles (GHUVs). Soft Matter 2017, 13, 627-637. [CrossRef] [PubMed]

4. Henderson, I.M.; Paxton, W.F. Salt, Shake, Fuse-Giant Hybrid Polymer/Lipid Vesicles through Mechanically Activated Fusion. Angew. Chem. Int. Ed. 2014, 53, 3372-3376. [CrossRef]

5. Hu, S.W.; Huang, C.Y.; Tsao, H.K.; Sheng, Y.J. Hybrid membranes of lipids and diblock copolymers: From homogeneity to rafts to phase separation. Phys. Rev. E 2019, 99, 012403. [CrossRef]

6. Lim, S.K.; de Hoog, H.P.; Parikh, A.N.; Nallani, M.; Liedberg, B. Hybrid, Nanoscale Phospholipid/Block Copolymer Vesicles. Polymers 2013, 5, 1102-1114. [CrossRef]

7. Magnani, C.; Montis, C.; Mangiapia, G.; Mingotaud, A.F.; Mingotaud, C.; Roux, C.; Joseph, P.; Berti, D.; Lonettia, B. Hybrid vesicles from lipids and block copolymers: Phase behavior from the micro- to the nano-scale. Colloids Surf. B Biointerfaces 2018, 168, 18-28. [CrossRef]

8. Nam, J.; Vanderlick, T.K.; Beales, P.A. Formation and dissolution of phospholipid domains with varying textures in hybrid lipo-polymersomes. Soft Matter 2012, 8, 7982-7988. [CrossRef]

9. Schulz, M.; Binder, W.H. Mixed Hybrid Lipid/Polymer Vesicles as a Novel Membrane Platform. Macromol. Rapid Commun. 2015, 36, 2031-2041. [CrossRef]

10. Schulz, M.; Glatte, D.; Meister, A.; Scholtysek, P.; Kerth, A.; Blume, A.; Bacia, K.; Binder, W.H. Hybrid lipid/polymer giant unilamellar vesicles: Effects of incorporated biocompatible PIB-PEO block copolymers on vesicle properties. Soft Matter 2011, 7, 8100-8110. [CrossRef]

11. Abraham, T.; Mao, M.; Tan, C.E.M. Engineering approaches of smart, bio-inspired vesicles for biomedical applications. Phys. Biol. 2018, 15, 061001. [CrossRef] [PubMed]

12. Bixner, O.; Bello, G.; Virk, M.; Kurzhals, S.; Scheberl, A.; Gal, N.; Matysik, A.; Kraut, R.; Reimhult, E. Magneto-Thermal Release from Nanoscale Unilamellar Hybrid Vesicles. Chemnanomat 2016, 2, 1111-1120. [CrossRef]

13. Cheng, Z.L.; Elias, D.R.; Kamat, N.P.; Johnston, E.D.; Poloukhtine, A.; Popik, V.; Hammer, D.A.; Tsourkas, A. Improved Tumor Targeting of Polymer-Based Nanovesicles Using Polymer-Lipid Blends. Bioconj. Chem. 2011, 22, 2021-2029. [CrossRef] [PubMed]

14. Khan, S.; McCabe, J.; Hill, K.; Beales, P.A. Biodegradable hybrid block copolymer-Lipid vesicles as potential drug delivery systems. J. Colloid Interface Sci. 2020, 562, 418-428. [CrossRef]

15. Mohammadi, M.; Taghavi, S.; Abnous, K.; Taghdisi, S.M.; Ramezani, M.; Alibolandi, M. Hybrid Vesicular Drug Delivery Systems for Cancer Therapeutics. Adv. Funct. Mater. 2018, 28, 1802136. [CrossRef]

16. Palivan, C.G.; Goers, R.; Najer, A.; Zhang, X.Y.; Car, A.; Meier, W. Bioinspired polymer vesicles and membranes for biological and medical applications. Chem. Soc. Rev. 2016, 45, 377-411. [CrossRef]

17. Panneerselvam, K.; Lynge, M.E.; Riber, C.F.; Mena-Hernando, S.; Smith, A.A.A.; Goldie, K.N.; Zelikin, A.N.; Stadler, B. Phospholipid-polymer amphiphile hybrid assemblies and their interaction with macrophages. Biomicrofluidics 2015, 9, 052610. [CrossRef]

18. Patil, S.S.; Kumbhar, D.D.; Manwar, J.V.; Jadhao, R.G.; Bakal, R.L.; Wakode, S. Ultrasound-Assisted Facile Synthesis of Nanostructured Hybrid Vesicle for the Nasal Delivery of Indomethacin: Response Surface Optimization, Microstructure, Stability. AAPS PharmSciTech 2019, 20, 97. [CrossRef]

19. Pippa, N.; Kaditi, E.; Pispas, S.; Demetzos, C. PEO-b-PCL-DPPC chimeric nanocarriers: Self-assembly aspects in aqueous and biological media and drug incorporation. Soft Matter 2013, 9, 4073-4082. [CrossRef]

20. Pippa, N.; Merkouraki, M.; Pispas, S.; Demetzos, C. DPPC: MPOx chimeric advanced Drug Delivery nano Systems (chi-aDDnSs): Physicochemical and structural characterization, stability and drug release studies. Int. J. Pharm. 2013, 450, 1-10. [CrossRef]

21. Robertson, J.D.; Yealland, G.; Avila-Olias, M.; Chierico, L.; Bandmann, O.; Renshaw, S.A.; Battaglia, G. pH-Sensitive Tubular Polymersomes: Formation and Applications in Cellular Delivery. ACS Nano 2014, 8, 4650-4661. [CrossRef] [PubMed]

22. Rideau, E.; Dimova, R.; Schwille, P.; Wurm, F.R.; Landfester, K. Liposomes and polymersomes: A comparative review towards cell mimicking. Chem. Soc. Rev. 2018, 47, 8572-8610. [CrossRef] [PubMed] 
23. Beales, P.A.; Khan, S.; Muench, S.P.; Jeuken, L.J.C. Durable vesicles for reconstitution of membrane proteins in biotechnology. Biochem. Soc. Trans. 2017, 45, 15-26. [CrossRef] [PubMed]

24. Khan, S.; Li, M.Q.; Muench, S.P.; Jeuken, L.J.C.; Beales, P.A. Durable proteo-hybrid vesicles for the extended functional lifetime of membrane proteins in bionanotechnology. Chem. Commun. 2016, 52, 11020-11023. [CrossRef] [PubMed]

25. Paxton, W.F.; McAninch, P.T.; Achyuthan, K.E.; Shin, S.H.R.; Monteith, H.L. Monitoring and modulating ion traffic in hybrid lipid/polymer vesicles. Colloids Surf. B Biointerfaces 2017, 159, 268-276. [CrossRef] [PubMed]

26. Seneviratne, R.; Khan, S.; Moscrop, E.; Rappolt, M.; Muench, S.P.; Jeuken, L.J.C.; Beales, P.A. A reconstitution method for integral membrane proteins in hybrid lipid-polymer vesicles for enhanced functional durability. Methods 2018, 147, 142-149. [CrossRef]

27. Jacobs, M.L.; Boyd, M.A.; Kamat, N.P. Diblock copolymers enhance folding of a mechanosensitive membrane protein during cell-free expression. Proc. Natl. Acad. Sci. USA 2019, 116, 4031-4036. [CrossRef]

28. Lee, J.C.M.; Bermudez, H.; Discher, B.M.; Sheehan, M.A.; Won, Y.-Y.; Bates, F.S.; Discher, D.E. Preparation, stability, and in vitro performance of vesicles made with diblock copolymer. Biotechnol. Bioeng. 2001, 73, 135-145. [CrossRef]

29. Nam, J.; Beales, P.A.; Vanderlick, T.K. Giant Phospholipid/Block Copolymer Hybrid Vesicles: Mixing Behavior and Domain Formation. Langmuir 2011, 27, 1-6. [CrossRef]

30. Garni, M.; Wehr, R.; Avsar, S.Y.; John, C.; Palivan, C.; Meier, W. Polymer membranes as templates for bio-applications ranging from artificial cells to active surfaces. Eur. Polym. J. 2019, 112, 346-364. [CrossRef]

31. Dao, T.P.T.; Brulet, A.; Fernandes, F.; Er-Rafik, M.; Ferji, K.; Schweins, R.; Chapel, J.P.; Schmutz, F.M.; Prieto, M.; Sandre, O.; et al. Mixing Block Copolymers with Phospholipids at the Nanoscale: From Hybrid Polymer/Lipid Wormlike Micelles to Vesicles Presenting Lipid Nanodomains. Langmuir 2017, 33, 1705-1715. [CrossRef]

32. Stoenescu, R.; Graff, A.; Meier, W. Asymmetric ABC-triblock copolymer membranes induce a directed insertion of membrane proteins. Macromol. Biosci. 2004, 4, 930-935. [CrossRef]

33. Otrin, L.; Marusic, N.; Bednarz, C.; Vidakovic-Koch, T.; Lieberwirt, I.; Landfester, K.; Sundmacher, K. Toward Artificial Mitochondrion: Mimicking Oxidative Phosphorylation in Polymer and Hybrid Membranes. Nano Lett. 2017, 17, 6816-6821. [CrossRef] [PubMed]

34. Du, J.Z.; O'Reilly, R.K. Advances and challenges in smart and functional polymer vesicles. Soft Matter 2009, 5, 3544-3561. [CrossRef]

35. Le Meins, J.F.; Schatz, C.; Lecommandoux, S.; Sandre, O. Hybrid polymer/lipid vesicles: State of the art and future perspectives. Mater. Today 2013, 16, 397-402. [CrossRef]

36. Armenante, P.M.; Kirpekar, A.K. Sterilization in the Pharmaceutical and Biotechnology Industry. In Handbook of Downstream Processing; Springer: Dordrecht, The Netherlands, 1997; pp. 261-308.

37. World Health Organisation. Methods of Sterilisation. In The International Pharmacopoeia; World Health Organization: Geneva, Switzerland, 2019; pp. 1-3.

38. Zuidam, N.J.; Lee, S.S.L.; Crommelin, D.J.A. Sterilization of Liposomes by Heat-Treatment. Pharm. Res. 1993, 10, 1591-1596. [CrossRef]

39. Jang, T.H.; Park, S.C.; Yang, J.H.; Kim, J.Y.; Seok, J.H.; Park, U.S.; Choi, C.W.; Lee, S.R.; Han, J. Cryopreservation and its clinical applications. Integr. Med. Res. 2017, 6, 12-18. [CrossRef]

40. Cuhadar, S.; Koseoglu, M.; Atay, A.; Dirican, A. The effect of storage time and freeze-thaw cycles on the stability of serum samples. Biochem. Med. 2013, 23, 70-77. [CrossRef]

41. Sykes, C. Time- and Temperature-Controlled Transport: Supply Chain Challenges and Solutions. Pharm. Ther. 2018, 43, 154 .

42. de Castro, M.D.; Garcia, J.L. Analytical freeze-drying. Tech. Instrum. Anal. Chem. 2002, 24, 11-41.

43. Khan, I.; Elhissi, A.; Shah, M.; Alhnan, M.; Ahmed, W. Liposome-Based Carrier Systems and Devices Used for Pulmonary Drug Delivery. In Biomaterials and Medical Tribology; Woodhead Publishing: Cambridge, UK, 2013; pp. 395-443.

44. Franzé, S.; Selmin, F.; Samaritani, E.; Minghetti, P.; Cilurzo, F. Lyophilization of Liposomal Formulations: Still Necessary, Still Challenging. Pharmaceutics 2018, 10, 139. [CrossRef]

45. Meng, F.; Engbers, G.; Feijen, J. Biodegradable polymersomes as a basis for artificial cells: Encapsulation, release and targeting. J. Control. Release 2005, 101, 187-198. [CrossRef] [PubMed] 
46. Goldbach, P.; Brochart, H.; Wehrlé, P.; Stamm, A. Sterile filtration of liposomes: Retention of encapsulated carboxyfluorescein. Int. J. Pharm. 1995, 117, 225-230. [CrossRef]

47. Drulyte, I.; Johnson, R.M.; Hesketh, E.L.; Hurdiss, D.L.; Scarff, C.A.; Porav, S.A.; Ranson, N.A.; Muench, S.P.; Thompson, R.F. Approaches to altering particle distributions in cryo-electron microscopy sample preparation. Acta Crystallogr. Sect. D Struct. Biol. 2018, 74, 560-571. [CrossRef] [PubMed]

48. Milne, J.L.S.; Borgnia, M.J.; Bartesaghi, A.; Tran, E.E.H.; Earl, L.A.; Schauder, D.M.; Lengyel, J.; Pierson, J.; Patwardhan, A.; Subramaniam, S. Cryo-electron microscopy-A primer for the non-microscopist. FEBS J. 2013, 280, 28-45. [CrossRef] [PubMed]

49. Rice, W.J.; Cheng, A.; Noble, A.J.; Eng, E.T.; Kim, L.Y.; Carragher, B.; Potter, C.S. Routine determination of ice thickness for cryo-EM grids. J. Struct. Biol. 2018, 204, 38-44. [CrossRef] [PubMed]

50. Sejwal, K.; Chami, M.; Baumgartner, P.; Kowal, J.; Mller, S.A.; Stahlberg, H. Proteoliposomes-A system to study membrane proteins under buffer gradients by cryo-EM. Nanotechnol. Rev. 2017, 6, 57-74. [CrossRef]

51. Karlsson, G. Thickness measurements of lacey carbon films. J. Microsc. Oxf. 2001, 203, 326-328. [CrossRef]

52. Kikuchi, H.; Carlsson, A.; Yachi, K.; Hirota, S. Possibility of heat sterilization of liposomes. Chem. Pharm. Bull. 1991, 39, 1018-1022. [CrossRef]

53. Mann, A.; Kiefer, M.; Leuenberger, H. Thermal sterilization of heat-sensitive products using high-temperature short-time sterilization. J. Pharm. Sci. 2001, 90, 275-287. [CrossRef]

(C) 2020 by the authors. Licensee MDPI, Basel, Switzerland. This article is an open access article distributed under the terms and conditions of the Creative Commons Attribution (CC BY) license (http://creativecommons.org/licenses/by/4.0/). 